\title{
KJIM
}

\section{Hepatic hemangioma presenting as a large cystic tumor}

Jin-Seon Park ${ }^{1}$, Gi-Ae Kim¹, Jae-Jun Shim ${ }^{1}$ Bum-Soo Kim², Seong Jin Park ${ }^{3}$, and Youn Wha Kim

Departments of ${ }^{1}$ Internal Medicine, ${ }^{2}$ General Surgery, ${ }^{3}$ Radiology, and ${ }^{4}$ Pathology, Kyung Hee University School of Medicine, Seoul, Korea
Received: March 26, 2020

Revised : April 18, 2020

Accepted: April 21, 2020

\section{Correspondence to}

Jae-Jun Shim, M.D.

Tel: +82-2-958-8155

Fax: +82-2-968-1848

E-mail:joyshim@khu.ac.kr

https://orcid.org/0000-0003-

2497-8663
A 62-year-old female chronic hepatitis

$B$ patient was referred to our clinic after an incidental cystic tumor in her liver was found on abdominal ultrasound. Her serum alanine aminotransferase level was $31 \mathrm{U} / \mathrm{L}$ and the hepatitis B virus DNA titer was $1,900 \mathrm{IU} / \mathrm{mL}$. An abdominal ultrasound revealed a hypoechoic cystic tumor measuring 8.2 $\times 7.2 \mathrm{~cm}$ in the lateral lobe of the liver. A computed tomography (CT) scan showed a large exophytic cyst in the
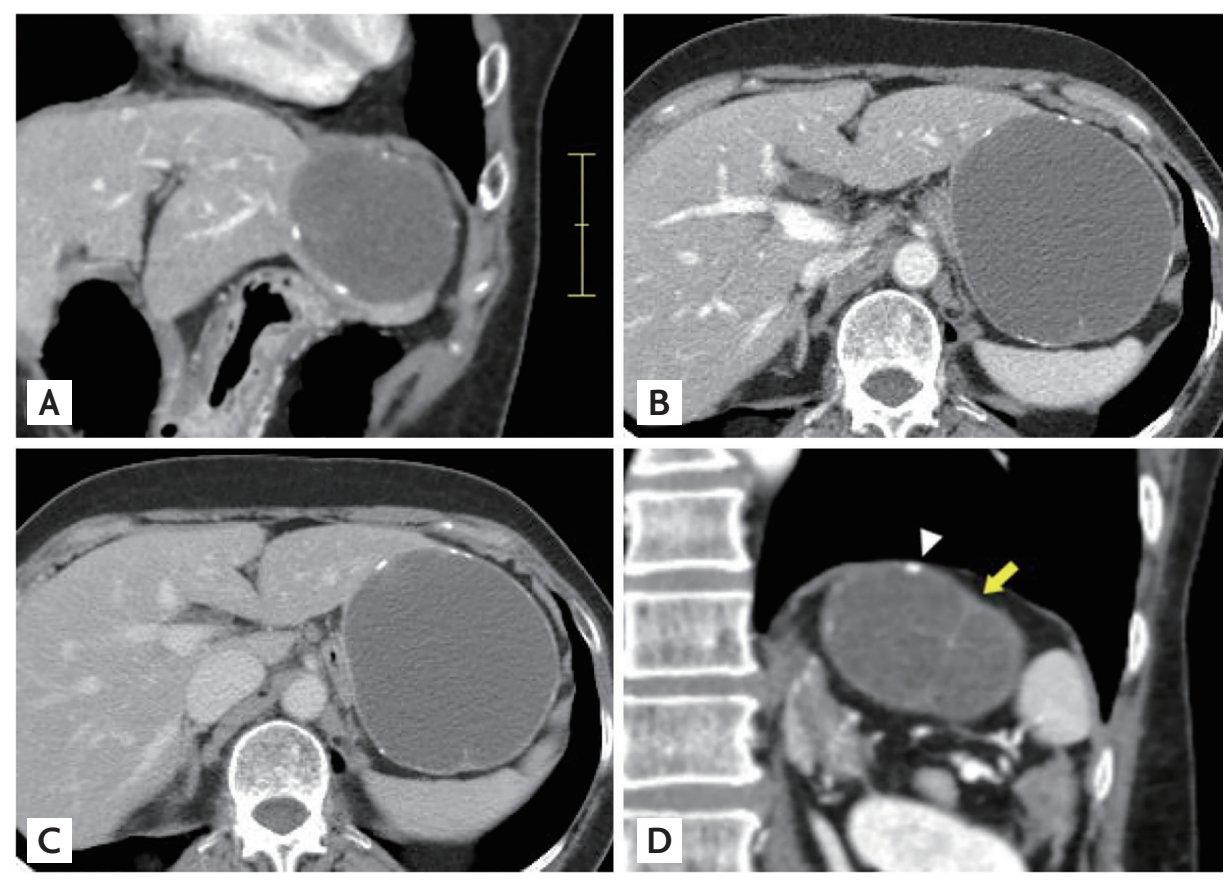

Figure 1. Computed tomography images of an exophytic hepatic hemangioma presenting as a large biliary cystadenoma. (A) Coronal image of the cyst from segment 2. (B, C) Transverse image of the tumor showing multiple fine calcifications in the rim. (D) A focal calcification (arrowhead) and thin septum (arrow). 

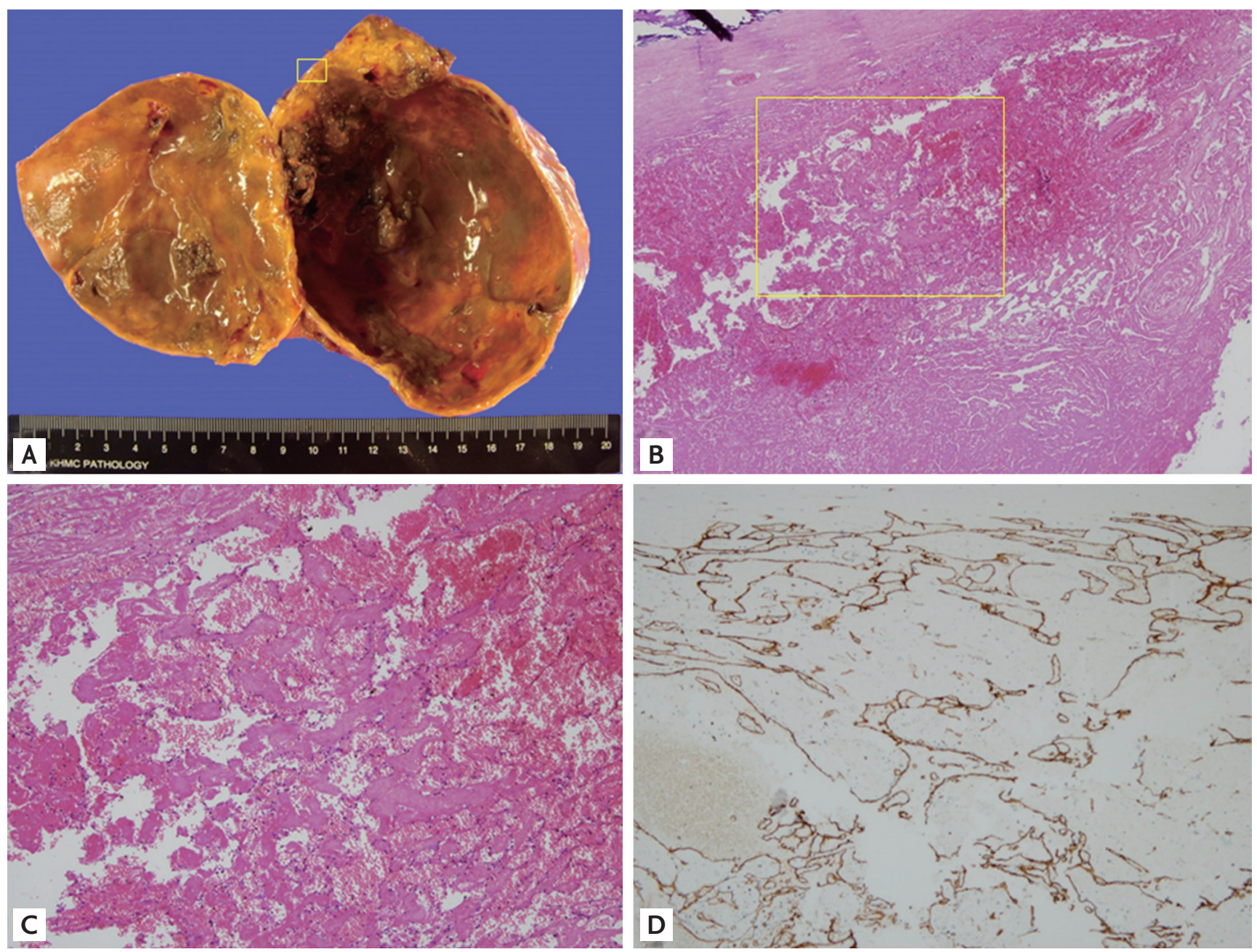

Figure 2. Histological view of the cystic tumor. (A) A macroscopic cut section showing a brownish round unilocular cyst with a dense fibrous wall. (B) Microscopic view of the small papillary lesion and cystic wall (the box in Fig. 2A, H\&E, ×40). (C) Mono-layered endothelial cells and fibrous stroma mixed with blood (the box in Fig. 2B, H\&E, ×100). (D) Immunohistochemical staining strong positivity for $\mathrm{CD}_{34}$, suggesting an endothelial origin (×100).

ic view of the cystic wall and small papillary remnants in that showed a dense fibrous wall without lining epithelium. The majority of the solid portion was almost completely necrotized and the remaining lesion had mono-layered endothelial cells and fibrous stroma mixed with blood cells, which indicated hepatic hemangioma (Fig. 2B and 2C). Immunohistochemical staining revealed strong positivity for cluster of differentiation 34 (CD34) and CD31 and negative for cytokeratin 19 (CK19), suggesting an endothelial origin (Fig. 2D). The cystic degeneration following the near-complete necrosis may have altered the presentation of this exophytic hemangioma into that of a cystic tumor.

Hepatic hemangiomas are the most common benign solid tumors in the liver. With ultrasound and CT scans becoming more accessible than ever, the detection of atypical hemangiomas mimicking malignant tumors has increased as well. As this case showed that a hepatic hemangioma can be misdiagnosed as a biliary cystic neoplasm. Thus, hepatic hemangiomas should be included in differential diagnoses of cystic tumors.

Written informed consent was obtained from the patient.

\section{Conflict of interest}

No potential conflict of interest relevant to this article was reported. 